\title{
Efficacy of Oral Alprazolam and Mefenamic Acid in Relieving Pain During Pan Retinal Photocoagulation
}

\author{
Muhammad Ali Haider ${ }^{1}$, Uzma Sattar ${ }^{2}$, Iftikhar Ahmed ${ }^{3}$ \\ Department of Ophthalmology, ${ }^{1}$ Al-Ehsan Eye Hospital, ${ }^{2,3}$ Rahbar Medical and Dental College, Lahore
}

\begin{abstract}
Purpose: To find out the efficacy of oral Alprazolam and Mefenamic acid in relieving pain during pan retinal photocoagulation in patients with proliferative diabetic retinopathy.
\end{abstract}

Study Design: Quasi experimental study.

Place and Duration of Study: Department of Ophthalmology, Al-Ehsan Eye Hospital, Lahore, from January 2018 to December 2018.

Methods: Patients were divided into two groups; groups A received one tablet of Xanax (Alprazolam $0.5 \mathrm{mg}$ ) and 2 tablets of Ponston Forte (Mefenamic acid $500 \mathrm{mg}$ ) an hour before PRP. In group B no pain relieving medicines were prescribed. Proparacaine hydrochloride $0.5 \%$ eye drops were instilled in the eye to achieve corneal anesthesia before the procedure in both the groups. Pain was scored, 15 minutes after PRP, using standard verbal rating scale and results were analyzed. Paired sample t-test was used to measure the mean difference in pain between the two groups. A p value of less than $0.01 \%$ was considered significant.

Results: Five hundred and sixty patients with proliferative diabetic retinopathy (PDR) requiring Pan Retinal Photocoagulation (PRP) were enrolled. There were 272 males and 288 females. Mean age of patients was $64.0 \pm$ 6.8 years. Mean pain score for group A was $4.0 \pm 0.6$ and for group B was $2.1 \pm 0.3$. Difference in pain score felt by each group was $-1.93 \pm 0.7$ with significant $p$ value of $\square 0.01 \%$.

Conclusion: Oral administration of Alprazolam and Mefenamic acid one hour before PRP in patients with proliferative diabetic retinopathy was effective in reducing pain and in attaining patient cooperation during the procedure.

Key Words: Pan Retinal Photocoagulation, Pain, Proliferative Diabetic Retinopathy, Alprazolam, Mefenamic acid.

How to Cite this Article: Haider MA, Sattar U, Ahmed I. Efficacy of Oral Alprazolam and Mefanamic Acid in Relieving Pain During Pan Retinal Photocoagulation. Pak J Ophthalmol. 2021, 37 (1): 38-42.

Doi: $10.36351 /$ pjo.v37i1.1084

Correspondence: Muhammad Ali Haider

Al-Ehsan Eye Hospital, Lahore

Email: alihaider_189@yahoo.com.

Received: June 19, 2020

Accepted: December 3, 2020

\section{INTRODUCTION}

Diabetic Retinopathy is one of the most prevalent forms of retinopathy affecting the diabetic patients in developed and undeveloped world. ${ }^{1}$ Life expectancy of diabetic patients is increasing with better medical provision, resulting in increased prevalence of diabetic retinopathy in the present world. This disease burden has many social and economic effects that have a far more serious impact in the under developed world because of limited human and technological resources to deal with the disease burden.,

The disease burden of diabetic retinopathy has increased many folds in recent years however early diagnosis and prompt treatment of patients is still a huge challenge. Recent advancements in Anti-VEGF 
therapy has revolutionized the treatment strategies for proliferative diabetic retinopathy, but PRP still has a major role in treatment. ${ }^{4-6}$ The procedure is less invasive and systemic risks are not involved as compared to Anti-VEGF administration. Since the advent of Argon laser numerous studies have been conducted to document the efficacy of treating proliferative diseases especially diabetic retinopathy. The Diabetic Retinopathy Study (DRS) and Early Treatment Diabetic Retinopathy Study (ETDRS) are landmark studies, documenting the effects of argon laser treatment in diabetic retinopathy.,8

Many factors like lack of patient education, limited treatment centers with proper equipment and financial constraints contribute to failure of patients in receiving proper laser administration. Compounding this problem is non-compliance on part of patients. One of the main reasons of failure to get proper argon laser sessions at our hospital for proliferative diabetic retinopathy was pain felt during the procedure. ${ }^{9,10}$

Numerous studies and trials have been conducted at various treatment facilities and various protocols have been devised to address this issue. It includes use of various common analgesics such Asentonox, Paracetamol, nonsteroidal anti-Inflammatory drugs (NSAIDs), Diazepam, and peri-bulbar anaesthesia. ${ }^{11-13}$ However, the most clinically effective analgesic is yet unknown.

In order to tackle the problem of non-compliance with argon laser treatment sessions secondary to pain, a protocol was established and applied to patients presenting at the retinal clinic at Al-Ehsan Eye Trust Hospital, Lahore. Retinal service at Al-Ehsan Eye Hospital, Lahore is faced with the challenge of increased patient burden, with an average daily turnover of 500 patients. A large proportion of the patients presenting in the service are diagnosed with diabetic retinopathy at various stages of evolution of the disease process. The scale of the problem is compounded by the patient's hesitation to undergo regular and timely sessions of PRP due to the pain encountered during the process. A protocol was devised to reduce the pain during the treatment process at the center ensuring completion of treatment sessions and better compliance.

The aim of the study was to document the intensity of pain and efficacy of pain relief after argon laser photocoagulation in patients with proliferative diabetic retinopathy, who followed the specified designed protocol at the hospital before undergoing laser application over the course of the study.

\section{METHODS}

This Quasi experimental study was conducted in the ophthalmology department of Al-Ehsan Eye Hospital, Lahore. Total 560 patients were enrolled over the course of one year from January 2018 to December 2018 after approval from hospital ethical committee. Diabetic patients (phakic or pseudophakic) without having any significant media opacity, diagnosed with proliferative diabetic retinopathy by retina consultant, based on fundus examination and fundus fluorescein angiography were included. Patients with any other associated ocular pathology such as neovascular glaucoma, media opacities or vitreous hemorrhage, history of prior argon treatment, or patients taking any pain medications for chronic systemic or ocular disorders and patients with cervical vertebral issues were excluded from the study. Informed consent was obtained. The relevant patient demographic profile, brief history, associated risk factors, stage of retinopathy and planned treatment were documented.

All the selected patients were divided into two groups by convenient sampling technique. Each group A and B consisted of 280 patients each. Patients were counseled before laser application about the procedure. Any drug allergy was documented. Pupillary dilation was achieved with $1 \%$ Tropicamide (1\% Mydriacyl, Alcon Laboratories) instilled every 15 minutes for an hour. In group A, one tablet of $0.5 \mathrm{mg}$ of Alprazolam (Xanax) and 2 tablets of Ponston Forte (Mefenamic acid $500 \mathrm{mg}$ ) were given orally one hour before the pan retinal photocoagulation procedure. In group B, no pain relieving medicine was given. Once the patients were ready for the procedure, $0.5 \%$ Proparacraine Hydrochloride (Alcaine, Alcon Novartis) eye drops were instilled in the eye twice with a 5-minute interval in-between before the procedure to anesthetize the cornea.

Treatment guidelines as described in DRS were followed. Argon laser was performed using Nidek argon green laser machine with the laser settings of wavelength $521 \mathrm{~nm}$, laser spot size $500 \mu \mathrm{m}$, repetitive mode with an exposure time of $0.1 \mathrm{sec}$. PRP 165 wide field lens was applied to the eye using methylcellulose gel as a coupling agent.

The laser power settings were adjusted to achieve moderate white burn one burn-width apart. A single 
surgeon performed the laser sessions in the retina clinic. Each session comprised of 1500 shots of laser. During laser application, a specific pattern was followed. Inferior half of retina was lasered first. Laser settings were tuned to the minimum possible when applying laser in the horizontal meridians. Fifteen minutes after the laser application, pain score was documented from each patient by using a standard verbal rating scale. This verbal scale included a range from 0 (no pain at all), 1 (slight discomfort), 2 (mild pain), 3 (moderate pain), 4 (severe pain), to 5 (extremely painful). All patients (group A and B) underwent the same set of standard protocol.

Data was recorded and analyzed by using SPSS version 22. Mean pain score was evaluated for both groups. Difference of pain score between two groups was evaluated by using paired sample t-test. A $p$ value of less than 0.05 was considered significant.

\section{RESULTS}

Out of total 560 patients, 272 were male and 288 were females. Mean age of patients was $64.0 \pm 6.8$ years. Mean pain score recorded in 'group A' was $2.1 \pm 0.3$ on standard verbal rating scale. In 'group B' the mean pain score recorded was $4.0 \pm 0.6$.

This table shows that most of the patients in 'group B' felt moderate to extreme pain. In 'group A' most of the patients felt pain at mild to moderate level ( $\mathrm{p}$ value 0.00 ). Details are depicted in table 1 .

Table 1: Mean of difference in pain between group $A$ and $B$.

\begin{tabular}{|c|c|c|c|c|c|c|c|c|}
\hline & \multicolumn{5}{|c|}{ Paired Differences } & \multirow[t]{3}{*}{$\mathbf{t}$} & \multirow[t]{3}{*}{ Df } & \multirow[t]{3}{*}{ Sig.(2-tailed) } \\
\hline & \multirow[t]{2}{*}{ Mean } & \multirow[t]{2}{*}{$\begin{array}{c}\text { Std. } \\
\text { Deviation }\end{array}$} & \multirow[t]{2}{*}{$\begin{array}{c}\text { Std. Error } \\
\text { Mean }\end{array}$} & \multicolumn{2}{|c|}{$\begin{array}{l}\text { 95\% Confidence Interval of } \\
\text { the Difference }\end{array}$} & & & \\
\hline & & & & Lower & Upper & & & \\
\hline Group A - Group B & -1.939 & .771 & .046 & -2.030 & -1.849 & -42.074 & 279 & .000 \\
\hline
\end{tabular}

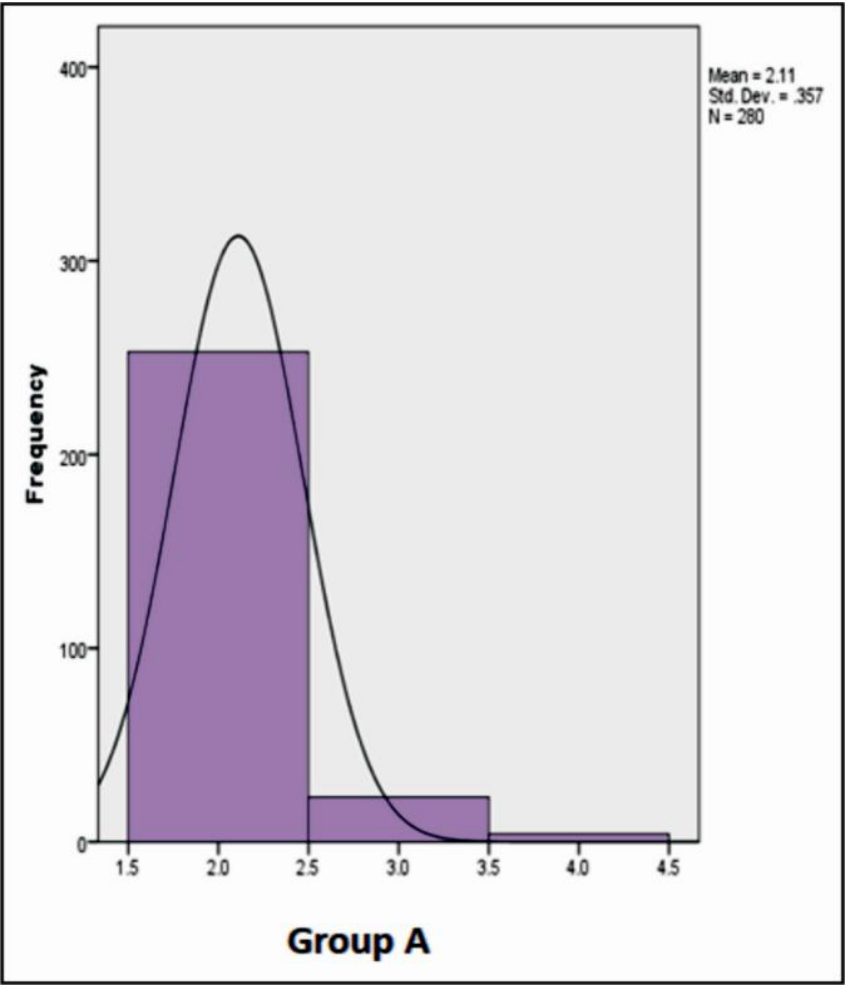

Graph 1: Showing pain score in Group A with distribution curve.

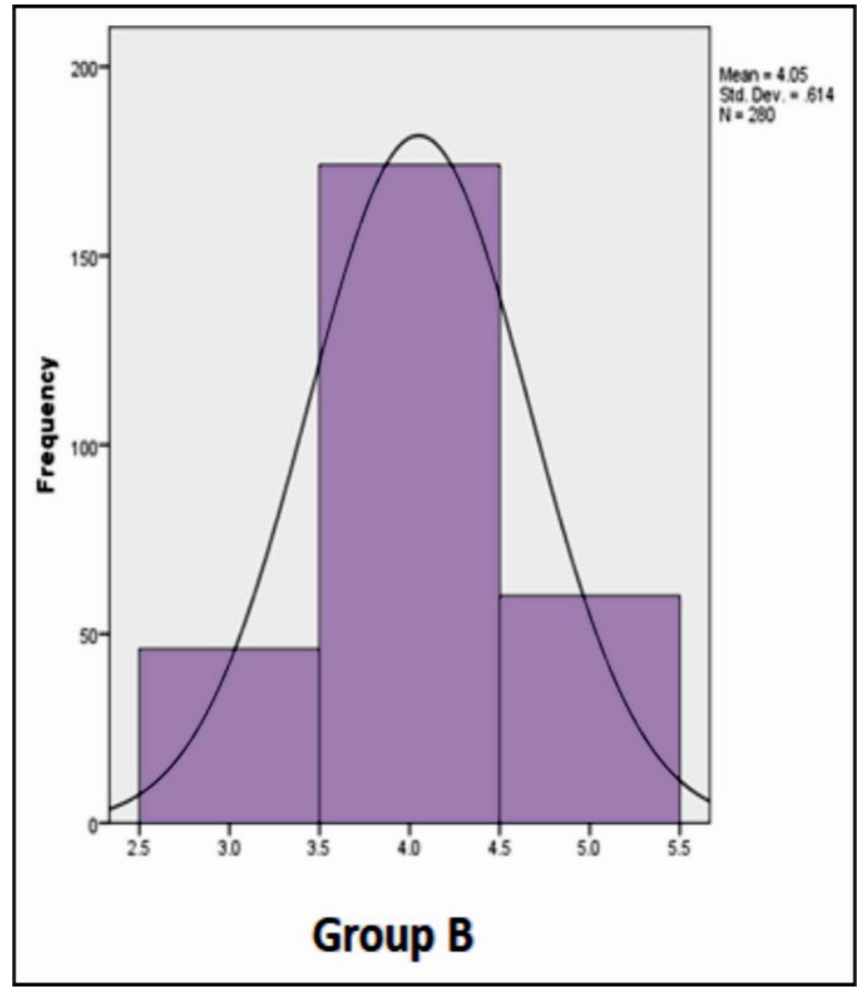

Graph 2: Showing pain score in Group B with distribution curve. 


\section{DISCUSSION}

Pan retinal photocoagulation is a relatively painful procedure and requires pre-emptive pain relief for patients to remain comfortable. Most units do not deliver pain relieving agents as shown in a national survey of all Ophthalmic Units within the NHS in UK. ${ }^{14}$ At other units, various methods are employed to control pain encountered by the patients during the procedure. We adopted a protocol that was noninvasive, cost effective, easily available, with least amount of side effects and easily applicable in our hospital settings and documented the results.

The results of our study are comparable to the studies conducted elsewhere. In a study conducted at Ophthalmology Service of the Hospital das Clínicas University of São Paulo Medical School by Rafael Barbosa de Araújo et al. They concluded that the use of $1000 \mathrm{mg}$ of Metamizole 40 minutes before PRP significantly reduced pain associated with the procedure in patients with PDR. ${ }^{15}$ Similarly, Hazem et al in their study reported lower degree of pain associated with a better hemodynamic response in patients given $150 \mathrm{mg}$ Pregabalin before photocoagulation. ${ }^{16}$

The laser energy is focused on the retinal tissue and is absorbed by the underlying retinal pigment epithelium (RPE) and choroid. ${ }^{17,18}$ Pigments within the RPE absorb laser energy and this is converted to thermal energy, raising tissue temperature, causing cellular death and coagulative necrosis. ${ }^{19}$ During laser application, the patients feel a pinching sensation with varying degrees of associated pain which is thought to arise from the photocoagulation of ciliary nerves running in the supra-choroidal space.

Pain is a product of higher brain center. It is a complex event with an emotional component and not just the appreciation of presence, location, and magnitude of nociceptive input. $^{20-22}$ Psychological factors can significantly influence the experience of pain. Fear and anxiety can enhance responses and interpretation of pain-producing events. Measures to reduce anxiety either with cognitive interventions or anxiolytic drugs can reduce pain. ${ }^{23}$

Limitation of this study is a single center trial. Placebo was not used in group B which could have psychological effect on the patients of this group.

\section{CONCLUSION}

Oral administration of Alprazolam (Xanax $0.5 \mathrm{mg}$ ) and 2 tablets of Ponstan Forte (Mefenamic acid $500 \mathrm{mg}$ ) one hour before pan retinal photocoagulation in patients with proliferative diabetic retinopathy was effective in reducing pain and in attaining patient cooperation during the procedure.

\section{Ethical Approval}

The study was approved by the Institutional review board/ Ethical review board. (EC Ref No: 01/18)

\section{Conflict of Interest}

Authors declared no conflict of interest

\section{REFERENCES}

1. Solomon SD, Chew E, Duh EJ, Sobrin L, Sun JK, Vander Beek BL, et al. Diabetic Retinopathy: A Position Statement by the American Diabetes Association. Diabetes Care, 2017; 40 (3): 412-418.

2. Stewart MW. Treatment of diabetic retinopathy: Recent advances and unresolved challenges. World J Diabetes, 2016; 7 (16): 333-341.

3. Baker CW, Jiang Y, Stone T. Recent Advancements in Diabetic Retinopathy Treatment from the Diabetic Retinopathy Clinical Research Network. Curr Opin Ophthalmol. 2016; 27 (3): 210-216.

4. The Diabetic Retinopathy Clinical Research N. Intravitreal Ranibizumab for Diabetic Macular Edema with Prompt versus Deferred Laser Treatment: 3-Year Randomized Trial Results. Ophthalmol. 2012; 119: 2312-2318.

5. Elman MJ, Ayala A, Bressler NM, Browning D, Flaxel CJ, Glassman AR, et al. Diabetic Retinopathy Clinical Research Network. Intravitreal Ranibizumab for diabetic macular edema with prompt versus deferred laser treatment: 5-year randomized trial results. Ophthalmology, 2015; 122 (2): 375-381.

Doi: 10.1016/j.ophtha.2014.08.047

6. Writing Committee for the Diabetic Retinopathy Clinical Research N. Pan retinal Photocoagulation vs. Intravitreous Ranibizumab for Proliferative Diabetic Retinopathy: A Randomized Clinical Trial. JAMA. 2015; 314 (20): 2137-2146.

7. Diabetic Retinopathy Study Research Group. Photocoagulation treatment of proliferative diabetic retinopathy: the second report of Diabetic Retinopathy Study (DRS) findings. Ophthalmology, 1978; 85: 82106.

8. Early Treatment of Diabetic Retinopathy Study Research Group. Early photocoagulation for diabetic retinopathy. ETDRS Report Number 9. Ophthalmology, 1991; 98 (Suppl.): 766-785. 
9. Hua W, Cao S, Cui J, Maberley D, Matsubara J. Analysis of reasons for noncompliance with laser treatment in patients of diabetic retinopathy. Can $\mathrm{J}$ Ophthalmol. 2013; 48 (2): 88-92.

10. Fong DS, Girach A, Boney A. Visual side effects of successful scatter laser photocoagulation surgery for proliferative diabetic retinopathy: a literature review. Retina, 2007; 27: 816-824.

11. Mirshahi A, Lashay A, Roozbahani M, Fard MA, Molaie S, Mireshghi M, et al. Pain score of patients undergoing single spot, short pulse laser versus conventional laser for diabetic retinopathy. Graefes Arch Clin Exp Ophthalmol. 2013; 251 (4): 1103-1107. Doi:10.1007/s00417-012-2167-5.

12. Inan UU, Polat $O$, Inan $S$, Yigit S, Baysal $Z$. Comparison of pain scores between patients undergoing pan retinal photocoagulation using navigated or pattern scan laser systems. Arq. Bras. Oftalmol. 2016; 79 (1): 15-18

13. Tesha PE, Giavedoni LR, Berger AR, Altomare F, Chow DR, Navajas EV, et al. Subconjunctival lidocaine before laser treatment: a randomized trial. Ophthalmology, 2010; 117 (9):1810-1814.

Doi: $0.1016 /$ J.Ophtha.2010.01.036.

14. Richardson $\mathbf{C}$, Waterman H. Pain relief during pan retinal photocoagulation for diabetic retinopathy: a national survey. Eye (Lond). 2009; 23 (12): 2233-2237.

15. De Araújo RB, Zacharias LC, de Azevedo BM. Giusti BS, Pretti RC, Takahashi WY, et al. Metamizole versus placebo for pan retinal photocoagulation pain control: a prospective doublemasked randomized controlled study. Int J Retin Vitr. 2015; 1: 21https://doi.org/10.1186/s40942-015-0021-8.

16. Hazem HA, Sayed JA. Efficacy of gabapentin versus pregabalin in pain control during and after pan retinal laser photocoagulation. J Egypt Ophthalmol Socie. 2014; 107: 28-32.

17. Hoeh AE, Pollithy S, Dithmar S. Factors affecting laser power in retinal Navilas laser treatment. Graefes Arch Clin Exp Ophthalmol. 2015; 253 (6): 849-854.

18. De Carlo TE, Bonini Filho MA, Baumal CR, Reichel E, Rogers A, Witkin AJ, et al. Evaluation of Preretinal Neovascularization in Proliferative Diabetic Retinopathy Using Optical Coherence Tomography Angiography. Ophthalmic Surg Lasers Imaging Retina, 2016; 47 (2): 115-119. Doi: 10.3928/232581602016;0126-03.
19. Ambresin A, Strueven V, Pournaras JA. Painless Indirect Argon Laser in High Risk Proliferative Diabetic Retinopathy. Klinische Monatsblätter Für Augenheilkunde. 2015; 232 (04): 509-513. Doi: $10.1055 / \mathrm{s}-0035-154579$.

20. Inan UU, Polat O, Inan S, Yigit S, Baysal Z. Comparison of pain scores between patients undergoing pan retinal photocoagulation using navigated or pattern scan laser systems. Arq Bras Oftalmol. 2016; 79 (1): 15-18. Doi: 10.5935/0004-2749.20160006.

21. Vujosevic S, Martini F, Longhin E, Convento E, Cavarzeran F, Midena E. Sub threshold micro pulse yellow laser versus sub threshold micro pulse infrared laser in center involving diabetic macular edema. Retina, 2015; 35 (8): 1594-1603. Doi: 10.1097/iae.0000000000000521-2015.

22. Chhablani J, Sambhana S, Mathai A, Gupta V, Arevalo JF, Kozak I. Clinical Efficacy of Navigated Pan retinal Photocoagulation in Proliferative Diabetic Retinopathy. Am J Ophthalmol. 2015; 159 (5): 884 889. Doi: 10.1016/j.ajo.2015.02.006.

23. Kaczkurkin AN. Cognitive-behavioral therapy for anxiety disorders: an update on the empirical evidence. Dialogues Clin Neurosci. 2015; 17 (3): 337-346.

\section{Author's Designation and Contribution}

Muhammad Ali Haider; Assistant Professor: Concepts, Design, Literature search, Data acquisition, Manuscript preparation.

Uzma Sattar; Investigative Occulist: Literature search, Data analysis, Statistical analysis, Manuscript preparation.

Iftikhar Ahmed; Professor: Literature search, Manuscript editing, Manuscript review. 